\title{
Risk of pneumonia in relation to body mass index in Australian Aboriginal people
}

\author{
D. T. PHUNG ${ }^{1,2 *}$ AND Z. WANG ${ }^{1}$ \\ ${ }^{1}$ Centre for Chronic Disease, School of Medicine, University of Queensland, Queensland, Australia \\ ${ }^{2}$ Centre for Environment and Population Health, Griffith University, Queensland, Australia
}

Received 6 December 2012; Final revision 29 January 2013; Accepted 19 February 2013

\section{SUMMARY}

This study examined the relationship between body mass index (BMI) and the risk of pneumonia in Aboriginal Australians. A total of 677 adults aged 20-60 years were followed up from the baseline examination during 1992-1995 to June 2012. The pneumonia events were identified through hospital records. Pneumonia incident rates were calculated according to BMI groups. Hazard ratios were computed using Cox regression adjusting for age, smoking and alcohol consumption status. The incident rate of pneumonia was $13 \cdot 3 / 1000$ person-years, and this rate was significantly higher in females than males (hazard ratio $=1 \cdot 5$ ). Compared to males with normal BMI $\left(18 \cdot 5-24.9 \mathrm{~kg} / \mathrm{m}^{2}\right)$, the adjusted hazard ratio was 3.5 for males with lowest BMI $(P<0 \cdot 01)$. Low BMI was significantly associated with a higher risk of hospitalized pneumonia for Aboriginal males. However, the U-shaped trend of this association indicates that the risk of pneumonia is likely to be associated with both low and high BMI.

Key words: Aboriginal people, body mass index, pneumonia, risk.

\section{INTRODUCTION}

Pneumonia is a significant cause of morbidity and mortality worldwide [1]. It has been demonstrated that hospitalizations with pneumonia have increased by $20-50 \%$ in Western populations during the last decade [2-4]. Some lifestyle factors such as cigarette smoking and alcohol consumption and health conditions such as heart disease, diabetes, chronic obstructive pulmonary disease and cancer are considered to be strong risk factors for pneumonia [2-9]. Nevertheless, there have been few studies about the relationship between obesity and the increased risk of pneumonia even though the prevalence of obesity has increased parallel to the pneumonia

\footnotetext{
* Author for correspondence: Dr D. T. Phung, 7 Angelina Street, Macgregor, Brisbane, QLD 4109, Queensland, Australia. (Email: d.phung@griffith.edu.au)
}

trend [10]. Moreover, the results of previous studies are inconsistent [1, 6, 11-13]. For instance, the increase in body mass index (BMI) was considered to be a protective factor for pneumonia by some authors $[6,12,13]$ but a risk in other studies $[1,11]$.

Respiratory disease is the third leading cause of death in Australian Aboriginal people, and mortality caused by respiratory diseases in Aboriginal people is three times higher than that of non-Aboriginal people [14]. Of respiratory diseases, pneumonia and influenza accounted for nearly $80 \%$ of hospitalizations in Aboriginal people, that is nearly a tenfold risk of hospitalization compared with non-Aboriginal people [15]. The lifestyle risk factors, including obesity, have been demonstrated to make significant contributions to chronic health conditions such as cardiovascular, renal diseases, and diabetes [14, 16-21]. However, no study has explored the relationship between obesity and pneumonia in Australian Aboriginal people. 
The objective of this study was to assess the association between BMI and the risk of hospitalized pneumonia in Aboriginal people in a remote community in the Northern Territory of Australia.

\section{METHODS}

\section{Study participants}

A prospective cohort study was conducted in a remote Aboriginal community in the Northern Territory of Australia. Baseline data on demographic, lifestyle, anthropometric measurements and biochemical markers of 1490 residents aged 2-76 years were collected from a community-wide screening conducted from 1992 to 1995 . For the purpose of this study, 698 adults aged 20-60 years were included, of these, 21 were excluded because they were known to have prior chronic medical conditions, which could have increased their risk of pneumonia, including heart disease (stroke, congestive heart failure), multiple sclerosis, Alzheimer's disease, AIDS, diabetes, cancer, asthma, and chronic obstructive pulmonary disease. A total of 677 participants (348 males, 329 females) were included in the final analysis.

Detailed baseline anthropometric measurements have been described previously [22]. Height was measured to the nearest $0.5 \mathrm{~cm}$ without shoes using a stadiometer. Weight was measured to the nearest $0.1 \mathrm{~kg}$ with the participants wearing light clothes only without shoes. BMI was defined as weight divided by height squared $\left(\mathrm{kg} / \mathrm{m}^{2}\right)$.

\section{Identification of pneumonia cases}

All participants were followed up to 30 June 2012. During the follow-up period, we identified pneumonia cases through hospital records according to ICD-9 (480-486) and ICD-10-AM codes (J12-J18). Hypostatic cases were not included (ICD-9: 514; ICD 10: $\mathrm{J} 18 \cdot 2$ ). Only the first-ever pneumonia incidents (fatal or non-fatal) during the follow-up period were included in the analysis to avoid using recurring cases from the same participant. For those participants who had pneumonia, the time of follow-up was counted from the date of their initial screening visit to the date of their first hospitalized admission with pneumonia. Patients who had been hospitalized for pneumonia before the study enrolment were excluded since BMI was measured after the occurrence of pneumonia. Participants who did not reach a pneumonia endpoint during the follow-up period were considered 'censored' at 31 May 2012.

\section{Data analysis}

Study participants were grouped according to BMI categories $\left(<18 \cdot 5,18 \cdot 5-24 \cdot 9,25-29 \cdot 9, \geqslant 30 \mathrm{~kg} / \mathrm{m}^{2}\right)$, similar to the categories used by the World Health Organization [23]. We used the date of the initial screening visit, the date of hospitalized admission with the first pneumonia event, date of death, and the end date of follow-up (30 June 2012) to compute the time at risk for each participant. The pneumonia incidence rates were calculated by dividing the number of first-ever pneumonia events by the total person-years of follow-up. The hazard ratios (HRs) with $95 \%$ confidence intervals (CIs) were computed using Cox's regression with BMI 18.5-24.9 as the reference group. The potential confounders, such as age (continuous), smoking status (yes/no) and alcohol consumption (yes/no) were adjusted for using Cox proportional hazards models. All analyses were performed using Stata v. 12.0 [24].

The project was approved by the Behavioural and Social Science Ethical Review Committee of the University of Queensland.

\section{RESULTS}

\section{Baseline characteristics of cohorts}

Of 677 participants, 81 males and 60 females had a first-ever pneumonia event during a mean follow-up time of 15.6 and 15.8 years, respectively. Of those 141 pneumonia cases, 23 were fatal. The baseline characteristics of those with and without pneumonia are shown in Table 1. Both males and females who developed pneumonia were older than those who did not develop pneumonia during the follow-up period. In terms of lifestyle factors, current smokers and alcohol drinkers appear to be more prevalent in nonpneumonia males; whereas, smoking was more prevalent in pneumonia than non-pneumonia females. In terms of anthropometric measurements, average weight, height, and BMI values were similar between pneumonia and non-pneumonia participants in both males and females.

\section{Incidence rates of pneumonia}

Overall, the incidence rate of first-ever pneumonia was $13 \cdot 3 / 1000$ person-years $(95 \%$ CI $11 \cdot 3-15 \cdot 7)$. This rate 
Table 1. Demographic data of the study cohort

\begin{tabular}{|c|c|c|c|c|c|}
\hline & \multicolumn{2}{|c|}{$\operatorname{Men}(N=348)$} & \multicolumn{2}{|c|}{ Women $(N=329)$} & \multirow{2}{*}{$\begin{array}{l}\text { Total } \\
(N=677)\end{array}$} \\
\hline & Pneumonia & Non-pneumonia & Pneumonia & Non-pneumonia & \\
\hline No. of subjects & 61 & 287 & 80 & 249 & 677 \\
\hline Age, mean (s.D.), years & $38 \cdot 1(9 \cdot 9)$ & $32 \cdot 3(9 \cdot 4)$ & $36 \cdot 1(11)$ & $34 \cdot 5(10)$ & $34 \cdot 1(10)$ \\
\hline Smoking status, Smoker, $n(\%)$ & $47(77 \cdot 1)$ & $242(84 \cdot 3)$ & $58(72 \cdot 5)$ & $160(64 \cdot 3)$ & $507(74 \cdot 9)$ \\
\hline $\begin{array}{l}\text { Alcohol consumption status, Drinker, } \\
n(\%)\end{array}$ & $50(82)$ & $250(87 \cdot 1)$ & $33(41 \cdot 3)$ & $82(32 \cdot 9)$ & $415(61 \cdot 3)$ \\
\hline Height, mean (s.D.), cm & $172 \cdot 2(6 \cdot 2)$ & $171 \cdot 3(6 \cdot 2)$ & $160(6 \cdot 4)$ & $161 \cdot 1(5 \cdot 5)$ & $166(8)$ \\
\hline Weight, mean (s.D.), kg & $70 \cdot 2(15 \cdot 2)$ & $67 \cdot 8(13 \cdot 2)$ & $65 \cdot 2(15 \cdot 7)$ & $64.9(16 \cdot 7)$ & $66 \cdot 6(15 \cdot 1)$ \\
\hline BMI, mean (S.D.), $\mathrm{kg} / \mathrm{m}^{2}$ & $23 \cdot 7(5 \cdot 1)$ & $23 \cdot 1(4 \cdot 1)$ & $25 \cdot 3(5 \cdot 7)$ & $25(6 \cdot 2)$ & $24 \cdot 1(5 \cdot 3)$ \\
\hline \multicolumn{6}{|l|}{ BMI group, $n(\%)$} \\
\hline$<18.5$ & $11(18 \cdot 0)$ & $33(11 \cdot 5)$ & $9(11 \cdot 3)$ & $41(16 \cdot 5)$ & $94(13 \cdot 9)$ \\
\hline $18 \cdot 5-24 \cdot 9$ & $27(44 \cdot 3)$ & $170(59 \cdot 2)$ & $33(41 \cdot 3)$ & $89(35 \cdot 7)$ & $319(47 \cdot 1)$ \\
\hline $25-29 \cdot 9$ & $15(24 \cdot 5)$ & $67(23 \cdot 3)$ & $23(28 \cdot 9)$ & $63(25 \cdot 3)$ & $168(24 \cdot 8)$ \\
\hline$\geqslant 30$ & $8(13 \cdot 1)$ & $17(5 \cdot 9)$ & $15(18 \cdot 8)$ & $56(22 \cdot 5)$ & $96(14 \cdot 2)$ \\
\hline
\end{tabular}

BMI, Body mass index.

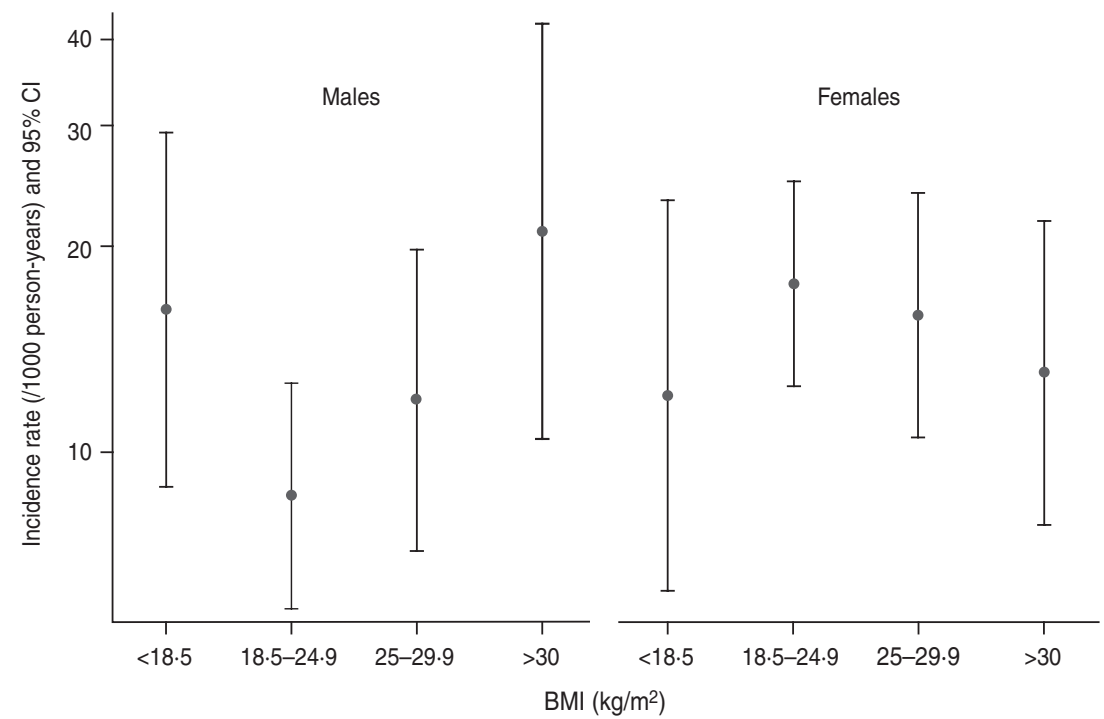

Fig. 1. Incidence rate of pneumonia against body mass index (BMI) in the Aboriginal cohort.

is similar to the Australian adjusted rate $(13 \cdot 2 / 1000$ person-years) in Australian Indigenous people reported by AIHW [16]. The rate for females (15.4/1000 person-years, $95 \%$ CI $12 \cdot 3-19 \cdot 1)$ was significantly higher than that for males $(11 \cdot 2 / 1000$ person-years, 95\% CI $8 \cdot 7-14 \cdot 5)$ with a HR of $1.5(95 \%$ CI $1-2, P<0 \cdot 03)$. The incidence rates of first-ever pneumonia by BMI group and gender are shown in Figure 1. For males, the highest incidence rate of pneumonia $(16 \cdot 2 / 1000$ person-years, 95\% CI 8.9-29.2) was seen in the lowest BMI group $\left(<18.5 \mathrm{~kg} / \mathrm{m}^{2}\right)$, and the lowest incidence rate $(8 \cdot 7 / 1000$ person-years; 95\% CI 5.9-12.6) in the normal BMI group $\left(18 \cdot 5-24 \cdot 9 \mathrm{~kg} / \mathrm{m}^{2}\right)$. The incidence rate increased with the increase of BMI in males $(11.9$ and $21 \cdot 1$, for BMI $25-29 \cdot 9$ and $>30 \mathrm{~kg} / \mathrm{m}^{2}$ groups, respectively). On the contrary, no such a trend of pneumonia incidence rates was observed in females.

\section{Risk factors of pneumonia}

Associations of age, smoking and alcohol consumption with pneumonia

For both men and women, age was a strong risk factor for pneumonia in the multivariate Cox model. A 1 -year increase in age resulted in a $4 \%$ and $5 \%$ increase 
Table 2. Hazard ratios (HRs) for hospitalized pneumonia by gender and body mass index (BMI)

\begin{tabular}{|c|c|c|c|c|}
\hline & \multicolumn{2}{|l|}{ Crude HR } & \multicolumn{2}{|l|}{ Adjusted HR* } \\
\hline & HR $(95 \%$ CI) & $P$ value & $\operatorname{HR}(95 \% \mathrm{CI})$ & $P$ value \\
\hline \multicolumn{5}{|l|}{ Male } \\
\hline BMI (kg/m²) & & $0 \cdot 08$ & & $<0 \cdot 01$ \\
\hline$<18 \cdot 5$ & $2 \cdot 2(1 \cdot 1-4 \cdot 6)$ & $0 \cdot 03$ & $3 \cdot 5(1 \cdot 6-7 \cdot 6)$ & $<0 \cdot 01$ \\
\hline $18 \cdot 5-24 \cdot 9$ & $1 \cdot 0$ (ref.) & & $1 \cdot 0$ (ref.) & \\
\hline $25-29 \cdot 9$ & $1 \cdot 6(0 \cdot 8-2 \cdot 9)$ & $0 \cdot 2$ & $1 \cdot 3(0 \cdot 7-2 \cdot 5)$ & $0 \cdot 3$ \\
\hline$\geqslant 30$ & $2 \cdot 1(0 \cdot 9-4 \cdot 7)$ & $0 \cdot 07$ & $1 \cdot 5(0 \cdot 7-3 \cdot 3)$ & $0 \cdot 4$ \\
\hline \multicolumn{5}{|l|}{ Female } \\
\hline BMI $\left(\mathrm{kg} / \mathrm{m}^{2}\right)$ & & $0 \cdot 72$ & & $0 \cdot 37$ \\
\hline$<18 \cdot 5$ & $0 \cdot 68(0 \cdot 32-1 \cdot 42)$ & $0 \cdot 3$ & $0.78(0.37-1.64)$ & $0 \cdot 5$ \\
\hline $18 \cdot 5-24 \cdot 9$ & $1 \cdot 0$ (ref.) & & $1 \cdot 0$ (ref.) & \\
\hline $25-29 \cdot 9$ & $0 \cdot 86(0 \cdot 5-1 \cdot 46)$ & $0 \cdot 6$ & $0.92(0.53-1.57)$ & $0 \cdot 8$ \\
\hline$\geqslant 30$ & $0 \cdot 79(0 \cdot 43-1 \cdot 46)$ & $0 \cdot 4$ & $0.87(0.46-1.64)$ & $0 \cdot 7$ \\
\hline
\end{tabular}

CI, Confidence interval.

* Adjusted for baseline age, smoking, and alcohol consumption status.

in the risk of pneumonia (men: HR $1 \cdot 05,95 \% \mathrm{CI}$ $1 \cdot 03-1 \cdot 09$; women: HR $1 \cdot 04$, 95\% CI $1 \cdot 02-1 \cdot 09$, $P<0 \cdot 01)$. Smoking is significantly associated with increased risk of pneumonia for men (HR 1.8, 95\% CI $\quad 0 \cdot 9-3 \cdot 4, \quad P<0 \cdot 05)$ whereas this association was not significant for women (HR $1 \cdot 03 ; 95 \%$ CI $0 \cdot 7-1 \cdot 5, P<0 \cdot 8)$. Alcohol consumption was positively associated with pneumonia in both men and women; however, the association was not statistically significant (men: HR 1.2, 95\% CI 0.6-2.4; women: HR $1 \cdot 2,95 \%$ CI $0 \cdot 8-1 \cdot 7, P>0 \cdot 1)$.

\section{BMI and the risk of pneumonia}

The crude and baseline adjusted HRs for hospitalized pneumonia according to BMI groups are presented in Table 2. The overall association between BMI and the risk of pneumonia was statistically significant in male participants $(P<0 \cdot 01)$ but not in female participants $(P=0 \cdot 37)$.

Compared to males with normal BMI $(18 \cdot 5-24 \cdot 9$ $\left.\mathrm{kg} / \mathrm{m}^{2}\right)$, the adjusted HR was $3.5(95 \%$ CI $1 \cdot 6-7 \cdot 6)$ with a statistical significance $(P<0 \cdot 01)$ for those with the lowest baseline BMI $\left(<18 \cdot 5 \mathrm{~kg} / \mathrm{m}^{2}\right)$. However, the HRs obtained from overweight and obese groups in males are $1.3(95 \% \mathrm{CI} 0.7-2.5)$ and $1.5(95 \% \mathrm{CI}$ $0 \cdot 7-3 \cdot 3$ ), respectively, but not statistically significant. The association was weaker, with adjusted HRs of 0.8 (95\% CI $0.4-1 \cdot 6)$ for overweight and $0.9(95 \%$ CI $0.5-1.6)$ for obese females. None of these were found to be statistically significant. The associations were different between crude and adjusted HRs, suggesting potential confounding by baseline age, smoking and alcohol consumption status. The association was stronger in males than in females.

\section{DISCUSSION}

Compared to previous studies, this is a long-term follow-up cohort study investigating the association between BMI and the risk of pneumonias. It is the first study of its kind in Australian Aboriginal people. Our results indicate that low BMI may play a role in the risk of pneumonia for male adults. Our findings are similar to the findings of major studies on this topic. A population-based case-control study by Almirall et al. [6] found an increased risk of community-acquired pneumonia (CAP) associated with low BMI in Spanish urban males. One large cohort study by Inoue et al. [12] illustrated that low BMI $\left(<18 \mathrm{~kg} / \mathrm{m}^{2}\right)$ was confirmed as a risk factor of pneumonia in both Japanese males and females aged 40-79 years. Similarly, a recent retrospective cohort study by Blumentals et al. [13] indicates that those who are underweight are at a higher risk of developing pneumonia; whereas being overweight (BMI, $25 \cdot 0-29 \cdot 9$ or $\geqslant 30 \mathrm{~kg} / \mathrm{m}^{2}$ ) was associated with a decreased pneumonia rate in British adults aged $18-70$ years [13].

In this study, we found that abnormal BMI (low, overweight, obesity) was more strongly related to the risk of pneumonia in males than in females. There are still no clear explanations about gender differences in the association between BMI and the risk of 
pneumonia in this study, and similar findings have been reported in previous studies $[1,11,13]$. Possible reasons for this include the difference in fat distribution, as abdominal obesity is more prevalent in males than in females, which can restrict the descent of the diaphragm and cause reduced ventilation at the lung bases [1]. Moreover, the differences in the prevalence of other factors of pneumonia such as smoking, alcohol consumption, physical activities may also play a role in modifying the association between males and females. For instance, the influence of physical activities related to respiratory infection was different between males and females [11, 25-27].

The U-shaped relationship between BMI and risk of pneumonia in Aboriginal males in this study is biologically plausible. Low BMI is associated with an increased risk of infection such as pneumonia, possibly due to malnutrition or an underlying illness $[28,29]$. On the other hand, obesity has also been suggested to cause an elevated risk of pneumonia because obesity was associated with impaired immune T- and/or B-cell function [30, 31]. Therefore, the results of this study were consistent with the evidence found in a cohort study conducted by Baik et al. [11] in the USA, which also reported a U-shaped relationship between BMI and immune dysfunction. Their results showed that not only the low BMI group but also those with BMI $>27 \mathrm{~kg} / \mathrm{m}^{2}$ had an increased risk of pneumonia compared to the reference group (BMI $21 \cdot 0-22 \cdot 9 \mathrm{~kg} / \mathrm{m}^{2}$ ) in men aged $44-75$ years.

The current study had several limitations. First, although some potential confounding factors were controlled for, residual confounding of these factors might have existed, since these were just measured as dichotomous variables (yes/no) rather than detailed amounts of cigarette smoking and alcohol consumption. For instance, Kornum et al. [1] indicated that the association between obesity and elevated pneumonia risk appeared to be strongest in non-smoking males. Moreover, during the long follow-up period, we did not monitor the changing status of smoking and alcohol consumption status. People who might have quit smoking and drinking were not taken into consideration in the analysis. Amirall et al. [32] found that ex-smokers who had ceased smoking for $>4$ years showed a statistically significant reduced risk of CAP compared to ex-smokers of $<1$ year. Second, even though participants who had chronic medical conditions, which could have caused a long hospitalization for those patients and an increased risk of pneumonia, were excluded from analyses, there was no data to examine whether pneumonia was a sequence of long hospitalization with chronic medical conditions or if it occurred at the same time as the medical condition. Similarly, we might have failed to exclude some cases who had chronic medical conditions but were admitted into hospital for a first-ever pneumonia event. Schnoor et al. [33] indicated that more than one respiratory infection during the previous year, chronic pulmonary diseases, number of comorbidities, and number of children in the household are independent risk factors for CAP. Third, those with minor pneumonia might not have visited the hospital, and therefore were not identified as pneumonia cases. Such a misclassification is likely to dilute the true association between BMI and pneumonia. Finally, the baseline data did not include information in change of physical weight and activities which could have confounded the relationship between BMI and pneumonia [11, 12]. Previous studies [25-27] indicated that physical activity is significantly associated with the risk of respiratory infection, including pneumonia.

In conclusion, the current study illustrates a possible relationship between low BMI and an elevated risk of pneumonia in Aboriginal adult males in the Northern Territory, Australia based on a long prospective cohort study. Our data do not support the association between BMI and the risk of pneumonia in female adults. Further studies with a larger sample size and better control for confounders are needed.

\section{ACKNOWLEDGEMENTS}

We thank the Aboriginal people who participated in this study. The baseline data were collected by the renal research team at the Menzies School of Health Research, Darwin, Australia. This project was supported by a National Health and Medical Research Council (NHMRC) project grant (APP1025350). Z.W. is supported by a NHMRC Research Fellowship (nos. 511013 and APP1042343).

\section{DECLARATION OF INTEREST}

None.

\section{REFERENCES}

1. Kornum JB, et al. Obesity and risk of subsequent hospitalization with pneumonia. European Respiratory Journal 2010; 36: 1330-1336. 
2. Fry AM, et al. Trends in hospitalizations for pneumonia among persons aged 65 years or older in the United State, 1988-2002. Journal of the American Medical Association 2005; 294: 2712-2719.

3. Thomsen RW, et al. Rising incidence and persistently high mortality of hospitalized pneumonia: a 10-year population-based study in Denmark. Journal of Internal Medicine 2006; 259: 410-417.

4. Trotter CL, et al. Increasing hospital admissions for pneumonia, England. Emerging Infectious Diseases 2008; 14: 727-733.

5. Lipsky BA, et al. Risk factors for acquired pneumonococcal infections. Archives of Internal Medicine 1986; 146: $2179-2185$.

6. Almirall $\mathbf{J}$, et al. Risk factors for community-acquired pneumonia in adults: a population-based case-control study. European Respiratory Journal 1999; 13: 349-355.

7. Paffenbarger RS, et al. Energy expenditure, cigarette smoking, and blood pressure level as related to death from specific diseases. In: Baik et al. 2000. A prospective study of age and lifestyle factors in relation to community-acquired pneumonia in US men and women. American Journal of Epidemiology 2000; 152: 264-171.

8. Fernadez-Sola $\mathbf{J}$, et al. High alcohol intake as a risk and prognostic factor for community-acquired pneumonia. Archives of Internal Medicine 1995; 155: 1649-1654.

9. Adams HG, Jordan C. Infections in the alcoholic. Medical Clinics of North America 1984; 68: 179-200.

10. Formiguera X, Canton A. Obesity: epidemiology and clinical aspects. Best Practice \& Research: Clinical Gastroenterology 2004; 18: 1125-1146.

11. Baik I, et al. A prospective study of age and lifestyle factors in relation to community-acquired pneumonia in US men and women. Archives of Internal Medicine 2000; 160: 3082-3088.

12. Inoue $\mathbf{Y}$, et $\boldsymbol{a l}$. Risk and protective factors related to mortality from pneumonia among middle-age and elderly community residents: the JACC study. Journal of Epidemiology 2007; 17: 194-202.

13. Blumentals WA, et al. Body mass index and the incidence of influenza-associated pneumonia in a UK primary care cohort. Influenza and Other Respiratory Viruses 2011; 6: 28-36.

14. Thomson N, et al. Overview of Australian Indigenous health status (http://healthinfonetecueduau/overview_ 2012pdf2011). Accessed 12 June 2012.

15. Plant AJ, Condon JR, Durling G. Northern Territory Department of Health and Community Services, 1995.

16. AIHW. Australian hospital statistics 2005-06. Health Services series no. 30, cat. no. HSE50. Canberra: Australian Institute of Health and Welfare, 2007.

17. Penm E. Cardiovascular disease and its associated risk factors in Aboriginal and Torres Strait Islander people 2004-5. AIHW cat. no. CVD41. Canberra: Australian Institute of Health and Welfare, 2008.

18. Hoy W, et al. Quantifying the excess risk for proteinuria, hypertension and diabetes in Australian
Aborigines: comparisons of profiles in three remote communities in the Northern Territory with those in the AusDiab study. Indigenous Health 2007; 31: $177-183$

19. Hoy WE, et al. Renal disease, the metabolic syndrome, and cardiovascular disease. Ethnicity and Disease 2006; 16: 2 .

20. Wang Z, Hoy W. Hypertension, dyslipidaemia, body mass index, diabetes and smoking status in Aboriginal Australians in a remote community. Ethnicity and Disease 2003; 13: 324-330.

21. Wang Z, Hoy WE. Waist circumference, body mass index, hip circumference and waist-to-hip ratio as predictors of cardiovascular disease in Aboriginal people. European Journal of Clinical Nutrition 2004; 58: 888893.

22. Wang Z, Hoy WE. Body size measurements as predictors of type 2 diabetes in Aboriginal people. International Journal of Obesity and Related Metabolic Disorders 2004; 28: 1580-1584.

23. NHLBI Obesity Education Innitiative. The practical guide: identification, evaluation and treatment of overweight and obesity in adults. National Institute of Health (NIH publication no. 00-4084), 2000.

24. StataCorp. Stata statistical software: release 12.0 . Colleage Station, Texas 77845 USA. 2011.

25. Nieman DC. Exercise, infection, and immunity. International Journal of Sports Medicine 1994; 15: S131S141.

26. Health GW, Macera CA, Nieman DC. Exercise and upper respiratory tract infection: isthere a relationship? Sports Medicine 1992; 14: 353-365.

27. Neuman MI, Willet WC, Curhan GC. Physical activity and the risk of community-acquired pneumonia in US women. American Journal of Medicine 2010; 123: 281. e287-281.e211.

28. LaCroix AZ, et al. Prospective study of pneumonia hospitalizations and mortality of U.S. older people: the role of chronic conditions, health behaviours, and nutritional status. Public Health Reports 1989; 104: 350-360

29. Saliva ME, et al. Disability and cognitive impairment are risk factors for pneumonia-related mortality on older adults. Public Health Reports 1993; 108: 314-322.

30. Tanaka S, et al. Impaired immunity in obesity: suppressed but reversible lymphocyte responsiveness. International Journal of Obesity 1993; 17: 631-636.

31. Gottschlich MM, et al. Significance of obesity on nutritional, immunologic, hormonal and clinical outcome parameters in burns. Journal of the American Dietetic Association 1993; 93: 1261-1268.

32. Amirall J, et al. New evidence of risk factors for community-acquired pneumonia: a population-based study. European Respiratory Journal 2008; 31: 1274-1284.

33. Schnoor M, et al. Risk factors for community-acquired pneumonia in German adults: the impact of children in the household. Epidemiology and Infection 2007; 135: 1389-1397. 